\title{
G

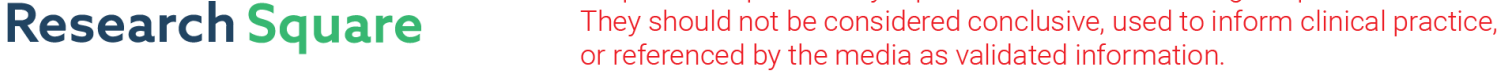 \\ Rheumatologists' understanding of Refractory Gout: A questionnaire survey in China
}

\section{Xinxin Han}

Peking Union Medical College Hospital

Yue Yin

Peking Union Medical College Hospital

\section{Yu Cao}

Peking Union Medical College Hospital

\section{Xiaotian Chu}

Peking Union Medical College Hospital

\section{Yingdong Han}

Peking Union Medical College Hospital

\section{Hong Di}

Peking Union Medical College Hospital

\section{$\mathrm{Na} \mathrm{Xu}$}

Peking Union Medical College Hospital

\section{Min Shen}

Peking Union Medical College Hospital

\section{Xuejun Zeng ( $\nabla$ zxjpumch@126.com )}

Peking Union Medical College Hospital https://orcid.org/0000-0002-6426-7586

\section{Yun Zhang}

Peking Union Medical College Hospital

\section{Research article}

Keywords: Gout, Diagnosis, Drug therapy, Refractory, Questionnaire

Posted Date: March 20th, 2020

DOI: https://doi.org/10.21203/rs.3.rs-18130/v1

License: (9) (1) This work is licensed under a Creative Commons Attribution 4.0 International License. Read Full License 


\section{Abstract}

\section{Background}

Refractory gout is a common problem faced by experienced rheumatologists, relevant research on the difficulties of gout treatment and the understanding of "refractory gout" by rheumatologists is lacking.

\section{Methods}

A total of 910 Chinese rheumatologists attending the annual academic conference on rheumatism completed the electronic questionnaires. The differences in the judgment of refractory gout were analyzed between the senior experience (SE) group and the general experience (GE) group.

Results

A total of $751(82.5 \%)$ respondents with relevant continuing medical education (CME) were included in our study, including 175 (23.3\%) SE rheumatologists and 576 (76.7\%) GE rheumatologists. Most of the rheumatologists $(715,95.2 \%)$ considered renal function insufficiency as a common complication making gout challenging to treat, while rheumatologists with different experiences had different attitudes towards obesity. For the chronic gouty arthritis patients with one or more tophi, $476(63.9 \%)$ Chinese rheumatologists preferred febuxostat as the first-line treatment option, while 582 (78.3\%) chose the maximum daily dose of $80 \mathrm{mg}$ for febuxostat, with a higher proportion in SE group than GE group. Meanwhile, the proportion of doctors who chose the dosage of all three uric-lowering drugs consistently with the guidelines was higher in the SE group. Moreover, 123 (70.3\%) people in the SE group thought that regardless of the use of drugs for prevention of attack during ULT, the recurrence of symptoms was symptomatic of refractory gout, while fewer in GE group agreed. Similarly, $119(68.0 \%)$ in the SE group thought that failure to achieve the targeted serum uric acid level within 6 months of standardized uratelowering therapy could be assigned to refractory gout, while fewer in GE group agreed. In terms of personal and social factors, the SE group had a higher percentage of physicians concerned about poor compliance leading to irregular treatment and drug abuse.

\section{Conclusions}

Most of the rheumatologists selected uric-lowering drugs and the maximum dosage of medicines consistently with guidelines. Uncontrolled symptoms, unattainable therapeutic targets, and unimproved lifestyle and compliance of patients are difficulties that Chinese rheumatologists have to face with during gout treatment.

\section{Background}

Gout, a crippling and highly prevalent disease, is a global health problem. In the year 2015, the global prevalence of gout ranged from $0.1-10 \%$ [1] and was about $1.1 \%$ in mainland China [2]. As these figures continue to climb, new research and guidelines are quickly emerging, furthering the understanding of gout 
both at home and abroad. Inevitably, clinicians encounter some problematic cases in their daily work. Currently, there is no universally accepted definition of 'refractory gout'. In 2011, ACR issued the diagnosis and management recommendations for gout and hyperuricemia, proposing that refractory gout refers to 1) inability to achieve a serum uric acid (SUA) level of $6.0 \mathrm{mg} / \mathrm{dL} ; 2$ ) occurrence of recurrent flares despite apparently adequate treatment; 3 ) patient presenting with persistent and/or extensive tophaceous disease [3]. However, later versions contained no reference to 'refractory gout'.

While refractory gout is a common problem faced by experienced rheumatologists, relevant research on the difficulties of gout treatment and the understanding of "refractory gout" by rheumatologists is lacking. Consequently, we conducted an in-depth investigation dedicated to the specific treatment of 'refractory gout'.

\section{Materials And Methods}

\section{Subjects}

A total of 910 rheumatologists participated in the annual academic conference on rheumatism in China between November 20th, 2019, and December 10th, 2019. All of the participants were informed on this survey before completing the anonymous questionnaire. Inclusion criteria required respondents to meet at least one of the following conditions: 1 ) rheumatologists with continuing medical education (CME) in gout; 2) rheumatologists who have studied the diagnosis and treatment guidelines for gout, including 2012 American College of Rheumatology (ACR) Guidelines for Management of Gout [4], 2018 EULAR Evidence-Based Recommendation: Diagnosis of Gout [5], 2018 Taiwan Multidisciplinary Consensus: Management of Gout and Hyperuricemia [6], 2016 Chinese Gout Diagnosis and Treatment Guide [7], 2017 Multidisciplinary expert consensus on diagnosis and treatment of hyperuricemia-related diseases in China [8], etc.

\section{Data Collection}

The questionnaire was designed by gout physicians and revised by four senior doctors in the field of gout research. A pilot study on ten physicians was done beforehand to analyze the questionnaire's validity. The questionnaire mainly included four aspects: 1) the general information of the doctors, including age, title, highest educational level, work unit, working years in the specialty, number of gout patients admitted annually. 2) Knowledge on the influences inducing complications and comorbidities in the treatment of gout, including tophi, joint deformity, obesity, kidney stone, hypertension, diabetes, malignancy, renal insufficiency, liver insufficiency, ischemic heart disease, history of allergy to urate-lowering drugs. 3) Knowledge of drug selection in refractory gout: including first-choice drugs, maximum daily dosage, and time required to achieve the target SUA concentration. 4) Knowledge of the concept of refractory gout: symptoms and signs, lab results, joint function, and other influencing factors. The online Questionnaire app in Chinese was used to collect primary and sample information for this study. 
Specialists were grouped into the senior experience (SE) group if they had more than 10 years of work experience and over 100 gout patients' annual encounters. Otherwise, they were grouped to the general experience (GE) group.

\section{Statistical analysis}

The preliminary data were summarized using IBM SPSS version 24.0 (IBM, Armonk, NY, USA) and Adobe Illustrator CC 2015(Adobe, Cal, USA). The qualitative data were expressed as percentages, and the quantitative data such as the age were calculated and analyzed using the mean and standard deviation. Student's t-test and Chi-square test were used to evaluate the general situation and selection rate difference between different groups. Using Chi-square goodness-of-fit test to analyze the differences among options in multiple topics. A p-value of 0.05 or less was defined as statistically significant.

\section{Results}

\section{General information}

Among 910 rheumatologists from 28 provinces (Fig. 1) who completed the questionnaire, 751 (82.5\%) were met the study criteria, including 175 (23.3\%) SE rheumatologists and 576 (76.7\%) GE rheumatologists. Compared with the GE group, the SE group was older (45.8 \pm 6.5 vs. $36.9 \pm 7.5)$, with a higher proportion of practicing in a tertiary medical institution (89.7\% vs. $79.9 \%)(P<0.001, P=0.001$, respectively). The percentage of refractory gout cases of $20 \%$ or more was more prominent in the SE group $(20.0 \%$ vs. $5.7 \%)$, while fewer claimed to only encounter $5 \%$ or fewer cases $(28.0 \%$ vs. $39.4 \%)(P<$ $0.001, P=0.007$, respectively). There was no significant difference in educational attainment between the SE group and the GE group $(P=1.000$; Table 1$)$.

\section{Influences of complications and comorbidities on the treatment of refractory gout}

In the opinion of rheumatologists, the most significant complication making gout difficult to treat was renal insufficiency $(95.2 \%)$, followed by tophi $(84.7 \%)$, allergic history of urate-lowering drugs $(83.6 \%)$, liver insufficiency $(80.3 \%)$, obesity $(76.2 \%)$, joint deformity $(73.9 \%)$, diabetes mellitus $(71.8 \%)$, kidney stone (69.6\%), ischemia heart disease (63.2\%), hypertension (60.6\%) and malignancy (57.1\%).

Compared with the GE group, the SE group has a lower proportion of physicians who consider obesity increases the difficulty of gout treatment $(68.0 \%$ vs. $78.6 \%, P=0.005$; Table 2$)$.

\section{Drug Selection In Refractory Gout}


For chronic tophaceous gout with no drug contraindication, 476 (63.9\%) doctors preferred febuxostat, while $162(21.7 \%)$ and 99 (13.3\%) specialists preferred allopurinol or benzbromarone. Upon selection of the maximum daily dosage, for allopurinol, $658(88.4 \%)$ chose $0.6 \mathrm{~g}$ or more, among whom $463(62.1 \%)$ chose $0.6 \mathrm{~g}$; for febuxostat, 582 (78.3\%) picked $80 \mathrm{mg}$; for benzbromarone, 509 (68.2\%) picked $100 \mathrm{mg}$. Overall, 411 (55.2\%) made dosage selections consistent with the ones recommended by relevant guidelines.

In SE group, a higher proportion of specialists chose $80 \mathrm{mg}$ as the maximum daily dose of febuxostat ( $84.6 \%$ vs $76.4 \%, P=0.021)$, and percentage of those who made all the right dosage selections was higher $(62.3 \%$ vs $53.0 \%, \mathrm{P}=0.037$; Fig. 2$)$

\section{Specialist's Opinion On The Concept Of "refractory Gout" Difficult to control symptoms}

A total of $730(97.2 \%)$ specialists thought that it was difficult to treat patients who still experience annual flares twice or more while on standardized urate-lowering therapy (ULT). Besides, 473 (63.0\%) specialists believed that recurrence of symptoms after initiation of ULT and preventive medications indicated refractory gout; $421(56.1 \%)$ specialists thought that symptoms of refractory gout could not be effectively controlled despite maximal NSAIDs administration; $313(41.7 \%)$ specialists considered they were dealing with refractory gout if there was symptomatic recurrence in the course of ULT titration starting from a small dose. Among them, $123(70.3 \%)$ SE thought that symptoms occurring upon preventive medication belonged to refractory gout, while $350(60.8 \%)$ GE agreed $(P=0.025$; Table 3$)$.

\section{Challenging to achieve SUA target}

A total of $392(52.2 \%)$ specialists considered that 3-6 months of standardized ULT failing to prompt SUA to target indicated difficult-to-treat gout. $71(10 \%), 209(27.8 \%)$, and $79(10.5 \%)$ specialists rated the time required to reach the target as less than 3 months, 6-12 months, and more than 12 months, respectively. $321(42.7 \%)$ specialists considered they were dealing with refractory gout if the serum uric acid could not drop below $6 \mathrm{mg} / \mathrm{dl}$ after monotherapy, while 457 (61\%) considered gout refractory if the tophus could not shrink after more than one year of standard ULT.

Furthermore, $68.0 \%$ of the SE thought that failure of SUA to achieve the target after standardized ULT up to 6 months was considered as difficult-to-treat gout, while $59.0 \%$ of GE agreed ( $P=0.034$; Table 3 ).

\section{Difficult to achieve a normal joint function}

Joint destruction (96.0\%), weak recovery of joints and surrounding muscles (63.0\%) and other comorbid inflammatory arthritis (67.9\%) were thought as the most important causes impeding functional improvement $(P<0.001)$, while comorbid osteoarthritis $(53.8 \%)$, traumatic injury $(28.5 \%)$, and inadequate 
rest $(26.5 \%)$ were considered the less important ones. 318 (42.3\%) specialists considered that all of the three main reasons might affect the recovery of joint function.

The GE was consistent with the SE on the reasons affecting the recovery of joint function (Fig. 3).

\section{Other factors making gout challenging to treat}

A total of $472(62.8 \%)$ specialists considered that the disease should be called refractory if patients' lifestyle and compliance failed to improve despite adequate education and regular ULT. Among the personal and social reasons for refractory gout, inadequate awareness of the disease (85.6\%), inadequate self-discipline on lifestyle improvement (89.2\%), poor compliance leading to irregular treatment $(92.3 \%)$ and failure to use medication reasonably $(72.6 \%)$ were the main reasons $(P<0.001)$. In addition, lack of long-term physician follow-up (57.3\%), limited therapeutic options (40.2\%), and a heavy financial burden (18.9\%) were the less important ones.

Compared with GE, the SE included a higher percentage of physicians concerned with poor compliance that was leading to irregular treatment and concerned about limited therapeutic options ( $96.0 \% \mathrm{vs.} 91.1 \%$, $47.6 \%$ vs. $37.7 \% ; P=0.035, P=0.011$; Table 4 ).

\section{Discussion}

The concept of "refractory gout" was first proposed in the study of benzbromarone in 1978, which suggested that benzbromarone was suitable for patients with refractory chronic gouty arthritis who were allergic to probenecid or allopurinol [9]. Since then, "refractory gout" has been frequently referenced; yet, never clearly defined. Refractory gout was described in the 2011 ACR recommendations for the diagnosis and management of gout and hyperuricemia, which proposed pegloticase as a potential treatment option [4]. However, because pegloticase is not suitable for patients with G6PD deficiency, and there are about 400 million patients with G6PD deficiency worldwide, the application of pegloticase is limited [10]. Since 2011, refractory gout has not been mentioned in new guidelines or studies. To the best of our knowledge, this is the first study that investigated refractory gout among specialists in China.

Research on gout has focused on the management of complications. The presence of complications narrows our therapeutic options and increases the difficulty of treatment. Chinese guidelines suggest glucocorticoids as the preferred treatment for the acute phase of gout in patients with moderate to severe renal insufficiency. It also suggests that the dosage of allopurinol in ULT should be tapered so as to lower down urate-lowering effect [8]. Patients with myocardial infarction and cardiac insufficiency should avoid the usage of cyclooxygenase 2 (COX-2) inhibitors during the acute attack of gout, while the febuxostat should be used with caution [11]. Patients with diabetes, hyperlipidemia, and hypertension should take an active approach to simultaneously lower glucose, lipid, and blood pressure, while liver and renal function may affect the use of drugs [6]. The 2016 EULAR recommendations for the diagnosis of gout underline the importance of screening and managing comorbidities frequently associated with gout[12]. According to the 2012 ACR Gout Guidelines, gout patients with more than four tophi palpable on the skin surface, 
rapid disease progression, and persistent severe arthritis are unanimously considered as having refractory gout [4]. Previous studies have shown that chronic gout patients with tophus had a longer course of the disease, more prominent joint swelling, higher disability index scores, and lower arthritis health index scores. Compared with gout patients without any tophus, physical function, and social function in patients with tophaceous gout were significantly declined, with reduced glomerular filtration rate, which increased the difficulty of treatment [13]. As a sign of refractory gout, the presence of any tophus has become the inclusion criteria for a variety of clinical trials of refractory gout drugs [14]. As confirmed in our study, Chinese specialists agree that comorbidities and complications increase the difficulty of gout treatment, especially renal insufficiency.

According to multiple guidelines, the first-line treatment for gout includes xanthine oxidase inhibitors allopurinol or febuxostat $[9,15]$, while the second-line treatment may include uricosuric agents such as benzbromarone or probenecid. 2011 ACR guidelines suggest that refractory gout patients should be treated with pegloticase [4]. In the 2016 EULAR guide, allopurinol is recommended as the first-line ULT with dose adjustment according to renal function. If the SU target cannot be achieved with allopurinol, then febuxostat, a uricosuric agent, or combination therapy of a xanthine oxidase inhibitor with a uricosuric agent should be considered. Pegloticase can be considered for patients with refractory gout [12]. In our survey, most physicians preferred xanthine oxidase inhibitor as urate-lowering drugs, specifically febuxostat. Allopurinol can induce adverse reactions such as Stevens-Johnson Syndrome (SJS) and Toxic Epidermal Necrolysis (TEN), while allopurinol-induced SJS/TEN has a strong and significant association between HLA-B*5801[16]. Therefore, HLA-B*5801 allele screening may be considered for patients treated with allopurinol that should be avoided in high-risk groups [4]. Allele screening increases the cost of drugs [17], reduces convenience, while some primary health care institutions do not have the ability to test for this allele. At present, the main form of allopurinol preparation in China is a $0.25 \mathrm{~g}$ sustained release tablet. All these factors lead to the limitation of allopurinol in China. However, febuxostat can make up for the above deficiencies, while the dosage of febuxostat in patients with mild to moderate renal insufficiency does not need to be adjusted, which makes it the first choice among Chinese specialists. In addition, $13 \%$ of specialists prefer benzbromarone as urate-lowing treatment, probably because more than $90 \%$ of hyperuricemia occurs due to decreased renal uric acid excretion [18]. In addition, benzbromarone is recommended by Endocrinology guidelines. The daily dose of allopurinol can be up to $800-900 \mathrm{mg}$ according to the international guidelines [15]; however, the maximum dose of allopurinol in the Chinese guidelines is $600 \mathrm{mg}$ per day. Therefore, we consider a daily dose of $600 \mathrm{mg}$ and above as a sufficient dose of allopurinol. The percentage of specialists whose opinion on the maximum dose of all three drugs was consistent with the official guidelines was significantly lower, and the percentage was higher in the SE, which indicated that the diagnosis and treatment experience, as well as continuing medical education experience, increased the understanding of the disease and urate-lowering drugs. In 2006, our team conducted a questionnaire survey on the diagnosis and treatment of gout among physicians at different levels finding that continuing medical education (CME) was the main factor for improvement of the diagnosis and treatment of gout [19]. Therefore, influenced by the length of continuing medical education, experienced 
physicians were more confident about the treatment difficulty caused by comorbidities and complications and more aware of the correct use of urate-lowering drugs.

In 2016, 88 rheumatologists with interest in gout from multiple countries established the preliminary remission criteria for Gout through Delphi. SUA level was identified as an important measurement to define the treatment target. The timeframe to achieve the SUA target was not established after 3 Delphi rounds, with 58\% choosing 6 months, 36\% choosing 1 year, and $6 \%$ choosing 3 months [20], which was consistent with our survey results. When asked how long it should take to achieve the target for refractory gout, $52 \%$ of the specialists thought $3-6$ months and $28 \%$ thought $6-12$ months. However, compared with the international panel of experts, our physicians expected a shorter timeframe to reach the target, so the "refractory gout" encountered by Chinese physicians might be attributable to that.

Fels E proposed that refractory gout describes patients with gout who suffer from persistent symptoms and inability to maintain the target serum uric acid levels $(<6 \mathrm{mg} / \mathrm{dl})$, which are usually associated with delayed or inadequate drug administration [21]. The inclusion criteria of clinical trials in pegloticase for refractory patients, required a baseline serum uric acid of $8.0 \mathrm{mg} / \mathrm{dL}$ or more and at least one of the following: 1) 3 or more self-reported gout flares during the previous 18 months; 2) 1 or more tophi; 3) gouty arthropathy, defined clinically or radiographically as joint damage due to gout. Patients also had contraindications to treatment with allopurinol or history of failure to normalize SUA despite at least three months of optimal allopurinol treatment (determined by the treating physician) [22]. Our findings are in line with the 2011 ACR guidelines:1) after the initiation of ULT, acute episodes occur despite the use of prophylactic drugs, or the titration of urate-lowering drugs from a small dose. Or maximal NSAIDs cannot control the symptoms effectively; 2 ) the serum uric acid cannot reach the standard with monotherapy; 3 ) tophus or tophi cannot shrink after more than one year of standard ULT. These guidelines are accepted by most physicians. Interestingly, many physicians believe that failure to improve lifestyle and compliance is also an important aspect of refractory gout. A British study that compared the nurse-led gout care and the usual care led by general practitioners (GPs) in the long-term treatment of gout patients over a period of two years found better compliance in gout patients, better treatment outcomes and lower costs under the nurseled gout care [23]. Therefore, the compliance of patients has a vital role in the treatment outcomes. Our team pointed out that currently, gout patients in our country are mainly treated in tertiary medical institutions. As the largest developing country, our primary health care system is not fully developed, and patient compliance directly leads to the changes in treatment outcome.

In the analysis of individual and social factors that lead to the difficulty of gout treatment, patients' compliance, self-discipline, and understanding of the disease were identified as important factors by all physicians. A survey on the medication compliance among gout patients found that up to $40 \%-50 \%$ of gout patients took medication irregularly [24], while $69.9 \%$ of gout patients in our country had poor compliance, which was often associated with poor prognosis [25]. Therefore, combined with our research results, we believe that patients' poor lifestyle and compliance were also important factors impacting the difficulty of gout treatment. In the previous randomized controlled trials, flare prophylaxis for up to 6 months during the initiation of ULT appeared to provide greater benefit than flare prophylaxis for 8 weeks, 
with no increase in adverse effects (AEs) [26]. The flare prophylaxis treatment determines whether the process of titration of urate-lowing treatment will go as planned and whether the patient can be regularly treated. Compared with the SE, a lower percentage of physicians in the GE consider recurrence of symptoms after initiation of ULT and preventive medications as being indicative of refractory gout. The difference between the two groups reveals that the higher experience was beneficial to the understanding of refractory gout.

Among the factors affecting the recovery of joint function in patients with refractory gout, joint destruction was the most recognized factor. The frequent onset of gout can cause joint damage, loss of function, and, eventually, disability [27] that has been widely recognized by physicians. Gout often needs to be differentiated from other crystal arthritis, especially pseudogout-calcium pyrophosphate arthritis [28]. However, in China, there are not so many tertiary medical institutions with the ability to perform arthrocentesis, thus making it so difficult to treat. Also, joint rehabilitation is a common concern among physicians. Previous studies have confirmed that long-term rehabilitation treatment in gout patients can significantly reduce the frequency of gout attacks [29]. Our team advocates rehabilitation exercises for gout patients and has begun comprehensive management of health education, joint rehabilitation, and drug therapy for patients.

Besides, there is a small population of gout patients who are challenging to treat due to genetic susceptibilities or some other predispositions [30]. This population usually requires a higher dose or a combined therapy along with a protracted treatment course, which may potentially pose even more difficulties; however, this entity is not within the scope of this study. The present study has some limitations. All the specialists included in the study had experience in gout diagnosis and treatment. They were all constantly continuing medical education, which is why knowledge level tests were not conducted on the study subjects.

\section{Conclusion}

Our results revealed that most of our specialists could correctly select urate-lowering drugs and had adequate knowledge of the maximum dosage. Uncontrolled symptoms, unattainable therapeutic effects, and unimproved lifestyle and compliance of patients resulted as major difficulties Chinese specialists were facing in gout treatment. Nevertheless, experience may account for the differences between the GE and the SE in the treatment of complications and comorbidities and the understanding of refractory gout.

\section{Abbreviations}

SE: senior experience; GE: general experience; CME: continuing medical education; ULT: urate-lowering therapy; SUA: serum uric acid; ACR: American College of Rheumatology; EULAR: The European League Against Rheumatism; NSAIDs: Nonsteroidal Anti-inflammatory Drugs; G6PD: glucose-6-phosphate dehydrogenease; SJS: Stevens-Johnson Syndrome; TEN: Toxic Epidermal Necrolysis; GPs: general practitioners; AEs: adverse effects. 


\section{Declarations}

\section{Ethics approval and consent to participate}

This study was approved by the Ethics Committee of Peking Union Medical College Hospital (S-K1088).

\section{Consent for publication!}

Not applicable.

\section{Availability of data and materials:}

All data analyzed in this paper is available from the corresponding author on reasonable request, and with appropriate additional ethical approvals, where necessary.

\section{Competing interests}

The authors declare that they have no competing interests.

\section{Funding}

This work was supported by National Natural Science Foundation of China 『Grand No. 81901667囚and Capital Medical Development Research Fund (2018-1-4012).

\section{Authors' contributions}

$\mathrm{XZ}, \mathrm{HX}$ and $\mathrm{YZ}$ participated in the conception and design of the work. $\mathrm{NX}$ and MS helped optimize the research and designed the questionnaire. $\mathrm{YY}, \mathrm{XC}, \mathrm{YH}$ and $\mathrm{HD}$ played key roles in collecting the data. $\mathrm{XH}$ made substantial contribution in data analysis and manuscript drafting. $Y C$ participated in writing the manuscript. $X Z$ and $Y Z$ revised the manuscript critically. All authors read and approved the final manuscript.

\section{Acknowledgments}

This study was supported by National Natural Science Foundation of China and Capital Medical Development Research Fund. We thank all the rheumatologists who participated in the questionnaire.

\section{References}

1. Kuo C F, Grainge M J, Zhang W, Doherty M. Global epidemiology of gout: prevalence, incidence and risk factors. Nat Rev Rheumatol 2015; 11: 649-662.

2. Liu R, Han C, Wu D, Xia X, Gu J, Guan H et al. Prevalence of Hyperuricemia and Gout in Mainland China from 2000 to 2014: A Systematic Review and Meta-Analysis. Biomed Res Int 2015; 2015: 762820 . 
3. Hamburger M, Baraf H S, Adamson T C, 3rd, Basile J, Bass L, Cole B et al. 2011 recommendations for the diagnosis and management of gout and hyperuricemia. Phys Sportsmed 2011;39: 98-123.

4. Khanna D, Fitzgerald J D, Khanna P P, Bae S, Singh M K, Neogi T et al. 2012 American College of Rheumatology guidelines for management of gout. Part 1: systematic nonpharmacologic and pharmacologic therapeutic approaches to hyperuricemia. Arthritis Care Res (Hoboken) 2012; 64: 1431-1446.

5. Richette P, Doherty M, Pascual E, Barskova V, Becce F, Castaneda J et al. 2018 updated European League Against Rheumatism evidence-based recommendations for the diagnosis of gout. Ann Rheum Dis 2020; 79: 31-38.

6. Yu K H, Chen D Y, Chen J H, Chen S Y, Chen S M, Cheng T T et al. Management of gout and hyperuricemia: Multidisciplinary consensus in Taiwan. Int J Rheum Dis 2018; 21: 772-787.

7. [2016 China gout clinical practice guideline]. Zhonghua Nei Ke Za Zhi 2016; 55: 892-899.

8. [Chinese multi-disciplinary consensus on the diagnosis and treatment of hyperuricemia and its related diseases]. Zhonghua Nei Ke Za Zhi 2017; 56: 235-248.

9. Yu T F. Pharmacokinetic and clinical studies of a new uricosuric agent - benzbromarone. J Rheumatol 1976; 3: 305-312.

10. Belfield K D, Tichy E M. Review and drug therapy implications of glucose-6-phosphate dehydrogenase deficiency. Am J Health Syst Pharm 2018; 75: 97-104.

11. Choi H, Neogi T, Stamp L, Dalbeth N, Terkeltaub R. New Perspectives in Rheumatology: Implications of the Cardiovascular Safety of Febuxostat and Allopurinol in Patients With Gout and Cardiovascular Morbidities Trial and the Associated Food and Drug Administration Public Safety Alert. Arthritis Rheumatol 2018; 70: 1702-1709.

12. Nuki G, Doherty M, Richette P. Current management of gout: practical messages from 2016 EULAR guidelines. Pol Arch Intern Med 2017; 127: 267-277.

13. Mandell B F, Yeo A E, Lipsky P E. Tophus resolution in patients with chronic refractory gout who have persistent urate-lowering responses to pegloticase. Arthritis Res Ther 2018; 20: 286.

14. Guttmann A, Krasnokutsky S, Pillinger M H, Berhanu A. Pegloticase in gout treatment - safety issues, latest evidence and clinical considerations. Ther Adv Drug Saf 2017; 8: 379-388.

15. Soskind R, Abazia D T, Bridgeman M B. Updates on the treatment of gout, including a review of updated treatment guidelines and use of small molecule therapies for difficult-to-treat gout and gout flares. Expert Opin Pharmacother 2017; 18: 1115-1125.

16. Somkrua R, Eickman E E, Saokaew S, Lohitnavy M, Chaiyakunapruk N. Association of HLA-B*5801 allele and allopurinol-induced Stevens Johnson syndrome and toxic epidermal necrolysis: a systematic review and meta-analysis. BMC Med Genet 2011; 12: 118.

17. Park D J, Kang J H, Lee J W, Lee K E, Wen L, Kim T J et al. Cost-effectiveness analysis of HLA-B5801 genotyping in the treatment of gout patients with chronic renal insufficiency in Korea. Arthritis Care Res (Hoboken) 2015; 67: 280-287. 
18. Dincer H E, Dincer A P, Levinson D J. Asymptomatic hyperuricemia: to treat or not to treat. Cleve Clin J Med 2002; 69: 594, 597, 600-592 passim.

19. Fang W, Zeng X, Li M, Chen L X, Schumacher H R, Jr., Zhang F. The management of gout at an academic healthcare center in Beijing: a physician survey. J Rheumatol 2006; 33: 2041-2049.

20. De Lautour H, Taylor W J, Adebajo A, Alten R, Burgos-Vargas R, Chapman P et al. Development of Preliminary Remission Criteria for Gout Using Delphi and 1000Minds Consensus Exercises. Arthritis Care Res (Hoboken) 2016; 68: 667-672.

21. Fels E, Sundy J S. Refractory gout: what is it and what to do about it? Curr Opin Rheumatol 2008; 20: 198-202.

22. Sundy J S, Baraf H S, Yood R A, Edwards N L, Gutierrez-Urena S R, Treadwell E L et al. Efficacy and tolerability of pegloticase for the treatment of chronic gout in patients refractory to conventional treatment: two randomized controlled trials. Jama 2011; 306: 711-720.

23. Doherty M, Jenkins W, Richardson H, Sarmanova A, Abhishek A, Ashton D et al. Efficacy and costeffectiveness of nurse-led care involving education and engagement of patients and a treat-to-target urate-lowering strategy versus usual care for gout: a randomised controlled trial. Lancet 2018; 392: 1403-1412.

24. Scheepers L, Van Onna M, Stehouwer C D A, Singh J A, Arts I C W, Boonen A. Medication adherence among patients with gout: A systematic review and meta-analysis. Semin Arthritis Rheum 2018; 47: 689-702.

25. Gao H, Chen L, Xu J, Yang F, Wang X, Liu D. [Compliance in intercriticalgout patients and its related factors]. Zhonghua Yi Xue Za Zhi 2015; 95: 2186-2189.

26. Wortmann R L, Macdonald P A, Hunt B, Jackson R L. Effect of prophylaxis on gout flares after the initiation of urate-lowering therapy: analysis of data from three phase III trials. Clin Ther 2010; 32: 2386-2397.

27. Lopez Lopez C O, Lugo E F, Alvarez-Hernandez E, Pelaez-Ballestas I, Burgos-Vargas R, VazquezMellado J. Severe tophaceous gout and disability: changes in the past 15 years. Clin Rheumatol 2017; 36: 199-204.

28. Schlee S, Bollheimer L C, Bertsch T, Sieber C C, Harle P. Crystal arthritides - gout and calcium pyrophosphate arthritis : Part 1: Epidemiology and pathophysiology. Z Gerontol Geriatr 2018; 51: 453-460.

29. Ivanova M M, Novoselova T M, Akimova T F, Tsurko V V. [Rehabilitation of patients with gout during outpatient follow-up]. Ter Arkh 1987; 59: 28-31.

30. Zhang Y, Wang D, Feng Y, Zhang W, Zeng X. Juvenile-onset gout and adipsic diabetes insipidus: A case report and literature review. J Int Med Res 2018; 46: 4829-4836.

\section{Tables}

Due to technical limitations, Tables 1-4 are provided in the Supplementary Files section. 


\section{Figures}

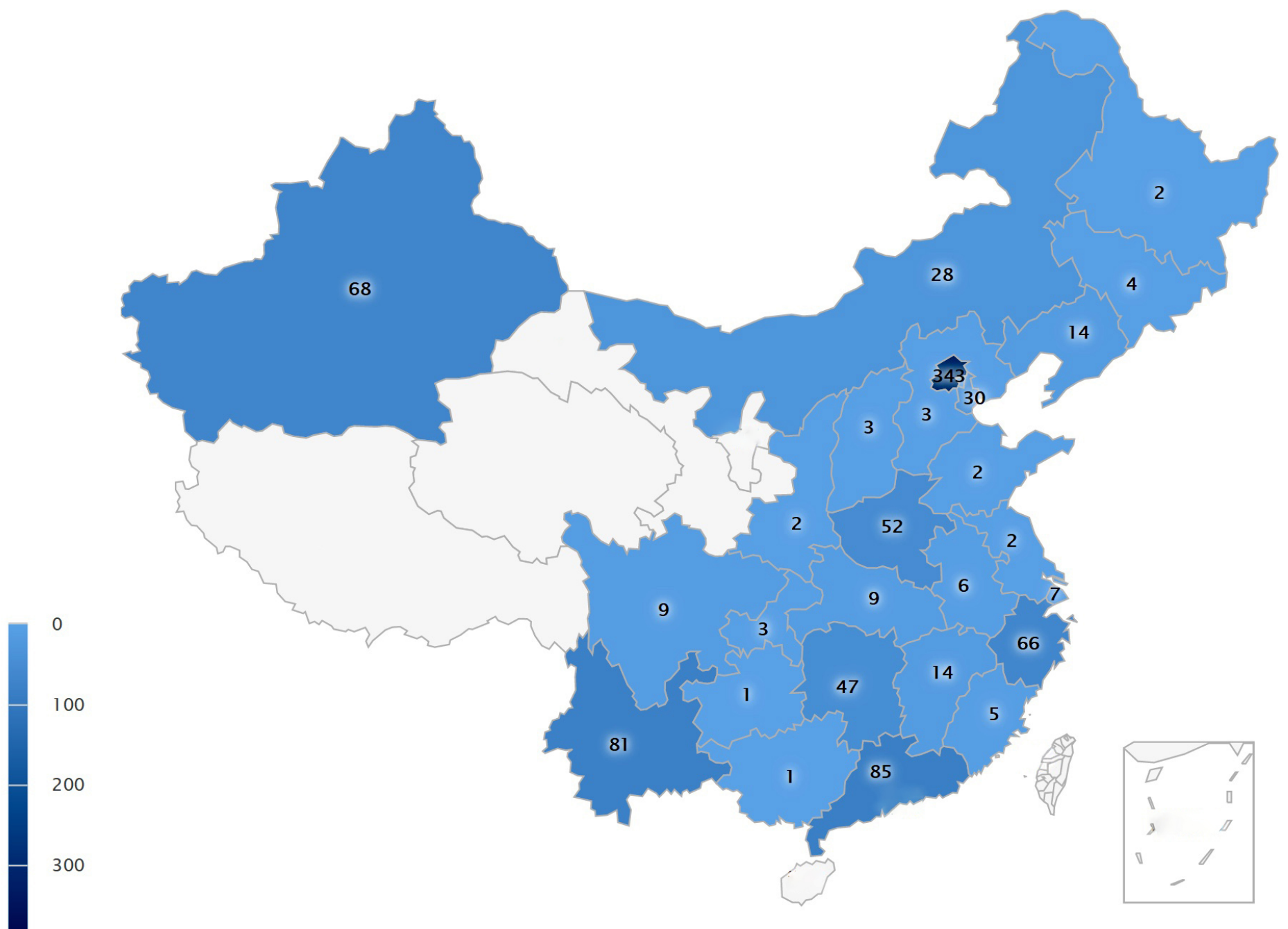

400

\section{Figure 1}

The distribution of respondents. Note: The designations employed and the presentation of the material on this map do not imply the expression of any opinion whatsoever on the part of Research Square concerning the legal status of any country, territory, city or area or of its authorities, or concerning the delimitation of its frontiers or boundaries. This map has been provided by the authors. 


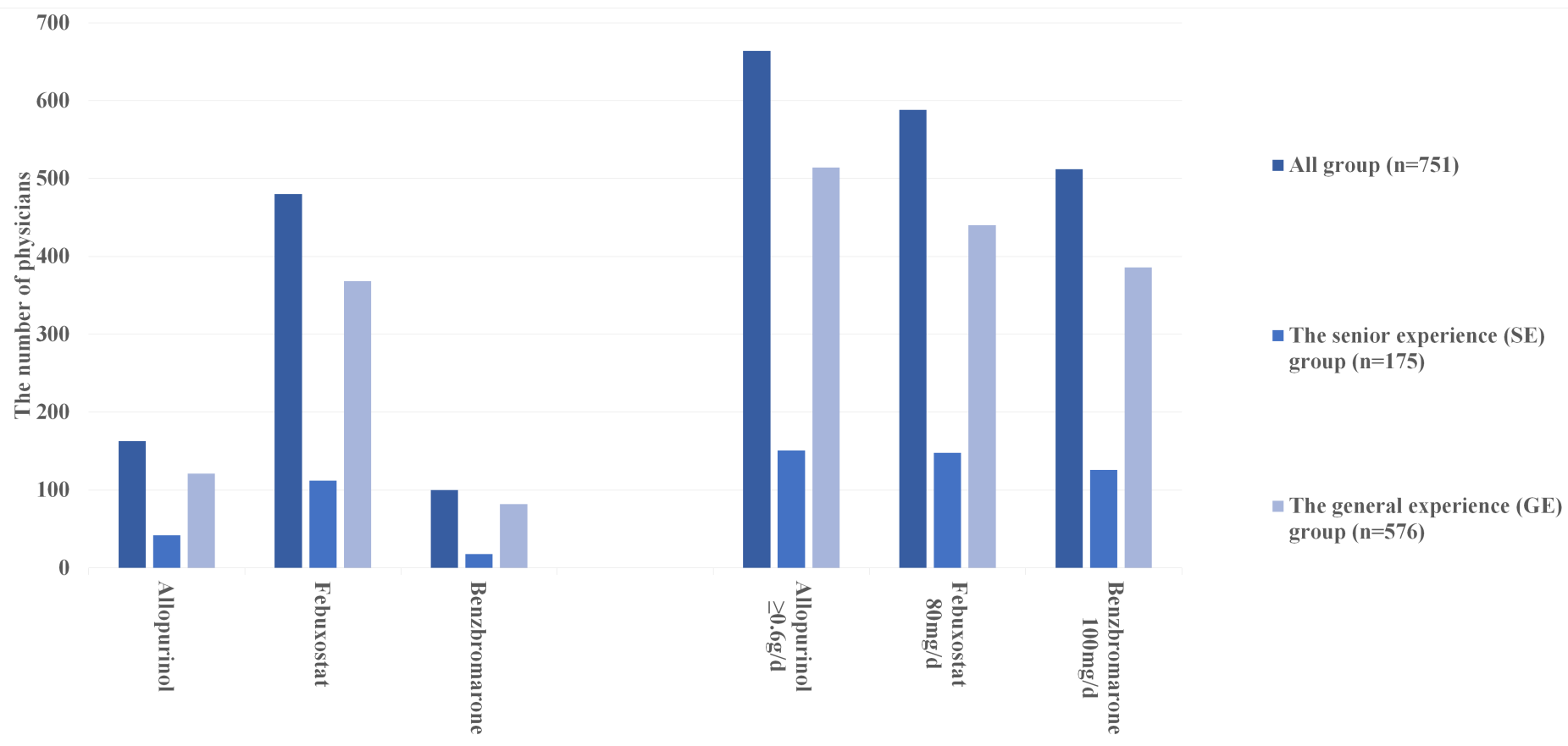

Figure 2

First-choice drugs and maximum daily dosage in refractory gout.

Inadequate rest

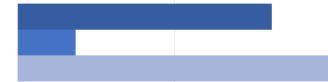

Traumatic injury

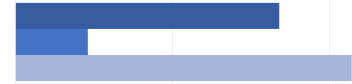

Comorbid osteoarthritis

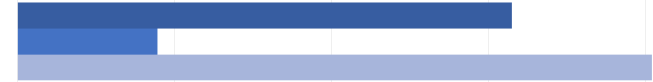

Poor recovery of joint and surrounding muscle function

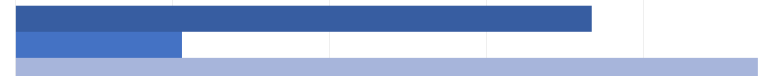

Other ongoing inflammatory arthritis

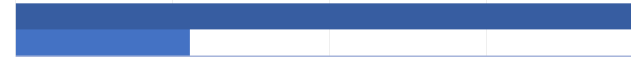

Joint destruction

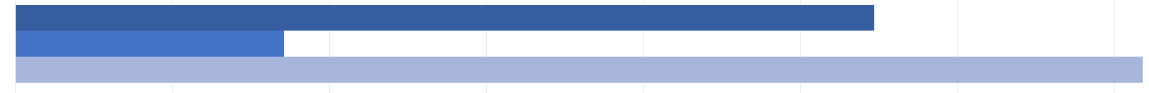

0

100

200

300

$400 \quad 500$

600

700

800

- The general experience $(\mathrm{GE})$ group $(\mathrm{n}=\mathbf{5 7 6})$

a The senior experience (SE) group $(n=175)$

All groups $(\mathrm{n}=\mathbf{7 5 1})$

\section{Figure 3}

The factors affecting the improvement of joint function in refractory gout patients after standardized treatment

\section{Supplementary Files}


This is a list of supplementary files associated with this preprint. Click to download.

- Table.pdf 